\title{
Prevalence and associations of lipodystrophy in HIV-infected outpatients in two London clinics
}

\author{
C. R. Loonam ${ }^{1}$, A. Duncan ${ }^{2}$, A. Mullen ${ }^{1}$ and B. S. Peters ${ }^{2}$ \\ ${ }^{1}$ Diabetes and Nutritional Sciences Division, King's College London, SE19NH and ${ }^{2}$ St Thomas' Hospital, Guy's and \\ St Thomas' NHS Foundation Trust, Westminster Bridge Road, SE1 7RH
}

Lipodystrophy (LD) associated with HIV and antiretroviral therapy (ART) is one of the most prevalent subtypes of acquired lipodystrophy ${ }^{1}$. Certain features of LD, such as abdominal obesity, are independently associated with cardiovascular disease in HIV ${ }^{2}$ There is a wide variation in the reported prevalence of LD in HIV, ranging from 9-83\%, which may in part reflect different definitions and assessment criteria ${ }^{3}$. We investigated the prevalence of LD and the factors associated with it among HIV-infected patients attending two urban UK outpatient clinics.

A retrospective analysis of data collected in 2005-2006 as part of the Cardiovascular Risk Evaluation and Antiretroviral Therapy Effects (CREATE) Study was conducted. A total of 257 patients with complete data were included in the analysis. LD was defined in the original study by the patient and their physician as any body fat abnormality other than general fat accumulation (lipoatrophy (LA) or lipohypertrophy (LH). Multivariable logistic regression analysis was used to determine factors associated with LD using SPSS version 20. Statistical significance was taken at $P<0.05$.

Thirty percent of patients had LD. Patients with LD had been diagnosed with HIV infection for longer $(P=0 \cdot 018)$, had higher CD4 cell counts $(P=0.001)$ and were more likely to have been on their current ART regimen for a longer duration $(P=0 \cdot 010)$ compared with those without LD. Patients with LD also had higher triglycerides $(P=0.026)$ and larger waist circumference $(P=0.014)$. The odds of developing LD was lower for males (odds ratio (OR) 0.31, $P=0.027$ ) and those receiving a first line regimen (OR 0.22, $P<0.001$ ), but higher for those with increasing CD4 count (OR 1.001, $P=0.034$ ), increased duration of ART (OR $1 \cdot 27, P=0.014)$, increased triglycerides (OR $1 \cdot 39, P=0.035)$ and increased fasting glucose (OR $1 \cdot 38, P=0 \cdot 030)$. No association was found between ethnicity and LD.

$\mathrm{LD}$ is a significant problem in this population and is associated with increased duration of ART, increasing CD4 count, triglycerides and glucose concentrations. These findings underscore the importance of screening and monitoring patients for signs of LD and highlight the need for studies to determine the current prevalence of LD among UK HIV-infected patients.

1. Garg A (2011) Lipodystrophies: Genetic and Acquired Body Fat Disorders. J Clin Endocrinol Metab. 96:3313-25.

2. Orlando G, Giovanni G, Stefano Z, et al. (2012) Ectopic Fat is Linked to Prior Cardiovascular Events in Men With HIV. JAIDS Journal of Acquired Immune Deficiency Syndromes. 59:494-7.

3. Loonam CR, Mullen A (2012) Nutrition and the HIV-associated lipodystrophy syndrome. Nutr Res Rev. 25:267-87. 